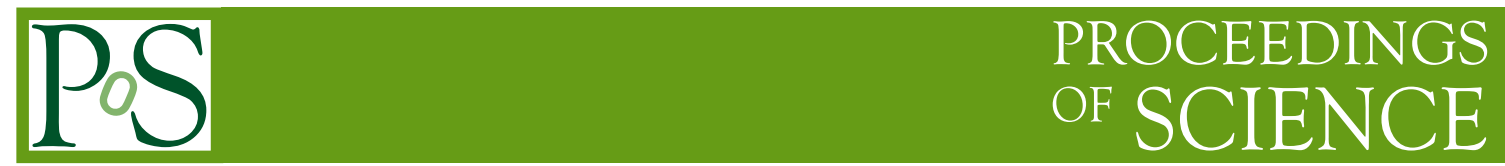

\title{
Reverse Engineering the Universe
}

\author{
Andrei Linde* \\ Author affiliation \\ Stanford Institute for Theoretical Physics and Department of Physics, Stanford University, \\ Stanford, CA 94305, USA E-mail: alinde@stanford.edu
}

\begin{abstract}
We describe the basic principles of inflationary cosmology and explain how it may help to create the universe, or maybe even the multiverse, from less than one milligram of matter. We review the recent observational evidence supporting this scenario.
\end{abstract}

2nd World Summit: Exploring the Dark Side of the Universe

25-29 June, 2018 - EDSU2018

University of Antilles, Pointe-à-Pitre, Guadeloupe, France

* Speaker. 


\section{Introduction: Hard art of the universe creation}

Suppose we want to create the universe suitable for life, and we want to do it in the simplest possible way. Is it possible to develop a fool-proof design? Let us look at our own universe at present, and then "play the movie back in time".

Some numbers are important here. The distance from Earth to the edge of the observable part of the universe is about 46.5 billion light years, or $4.4 \times 10^{28} \mathrm{~cm}$, in any direction. It contains about $10^{90}$ elementary particles. The total mass is about $10^{50}$ tons.

In quantum gravity it is very convenient to use system of units where $c=\hbar=M_{p}=1$. In these units, the density of matter in the expanding universe was $\rho=1 / t^{2}$. At $t<1$, density was $>O(1)$, and quantum fluctuations were too strong. The time $t=1$ (or, equivalently, $10^{-43}$ seconds, in more conventional units) is called the Planck time, and the density equal to 1 (or $10^{94} \mathrm{~g} / \mathrm{cm}^{3}$ ) is called the Planck density. At that time, each part of the universe of size $O(1)$ (Planck length $10^{-33}$ $\mathrm{cm})$ contained $O(1)$ particles, each of them with kinetic energy $O(1)$ in Planck units. One can talk about classical space - time only at $t>1$ and at density smaller than the Planck density.

According to the standard hot Big Bang universe, the total number of particles during its expansion did not change much, so the universe at the Planck time was supposed to contain about $10^{90}$ particles. If we calculate the total mass/energy of all particles that we can see now in the observable part of the universe, then at the Planck time it was about 30 orders of magnitude than now, i.e. about $10^{80}$ tons. This is simply because the wavelength of each particle was much shorter at that time, and its energy was much greater. The simplest way to estimate it is to take a typical energy of a particle at the Planck time, which is Planck energy $\sim 10^{-5} \mathrm{~g}$, and multiply it by the number of particles $10^{90}$. This gives $10^{85} \mathrm{~g}$, or $10^{79}$ tons. One may wonder where did this mass come from?

At the Planck time $t=O(1)$, there was one particle per Planck length $c t=O(1)$. Thus, at the Planck time $t=1$, the whole universe consisted of $10^{90}$ causally connected parts of size $c t=O(1)$. Such parts did not know about each other. If someone wanted to create the universe at the Planck time, he/she could only make a Very Small Bang in his/her own tiny part of the universe of a Planck size $c t=O(1)$. Everything else was beyond causal control. This means that if anybody would try to create a universe like ours in a hot Big Bang, he/she would be unable to create more than $10^{-90}$ of the part of the universe that we see now. Then what was responsible for the uniformity of the universe?

Is it possible to start with less than a milligram of matter (Planck mass), in a tiny speck of space of Planck size $\mathrm{O}(1)$, produce $10^{90}$ particles from it, and make the universe homogeneous and isotropic? As we will see, the answer to this question is positive, but only if one gives up many old ideas about the origin of the universe.

\section{Inflation to the rescue}

Consider the simplest model of a scalar field $\phi$ with a mass $m$ and with the potential energy density $V(\phi)=\frac{m^{2}}{2} \phi^{2}$. Since this function has a minimum at $\phi=0$, one may expect that the scalar field $\phi$ should oscillate near this minimum. This is indeed the case if the universe does not 
expand, in which case equation of motion for the scalar field coincides with equation for harmonic oscillator, $\ddot{\phi}=-m^{2} \phi$.

However, because of the expansion of the universe with Hubble constant $H=\dot{a} / a$, an additional term $3 H \dot{\phi}$ appears in the harmonic oscillator equation:

$$
\ddot{\phi}+3 H \dot{\phi}=-m^{2} \phi
$$

The term $3 H \dot{\phi}$ can be interpreted as a friction term. The Einstein equation for a homogeneous universe containing scalar field $\phi$ looks as follows:

$$
\left.H^{2}+\frac{k}{a^{2}}=\frac{1}{6}\left(\dot{\phi}^{2}+m^{2} \phi^{2}\right)\right) .
$$

Here $k=-1,0,1$ for an open, flat or closed universe respectively.

If the scalar field $\phi$ initially was large, the Hubble parameter $H$ was large too, according to the second equation. This means that the friction term $3 H \dot{\phi}$ was very large, and therefore the scalar field was moving very slowly, as a ball in a viscous liquid. Therefore at this stage the energy density of the scalar field, unlike the density of ordinary matter, remained almost constant, and expansion of the universe continued with a much greater speed than in the old cosmological theory. Due to the rapid growth of the scale of the universe and a slow motion of the field $\phi$, soon after the beginning of this regime one has $\ddot{\phi} \ll 3 H \dot{\phi}, H^{2} \gg \frac{k}{a^{2}}, \dot{\phi}^{2} \ll m^{2} \phi^{2}$, so the system of equations can be simplified:

$$
H=\frac{\dot{a}}{a}=\frac{m \phi}{\sqrt{6}}, \quad \dot{\phi}=-m \sqrt{\frac{2}{3}} .
$$

The first equation shows that if the field $\phi$ changes slowly, the size of the universe in this regime grows approximately as $e^{H t}$, where $H=\frac{m \phi}{\sqrt{6}}$. This is the stage of inflation, which ends when the field $\phi$ becomes much smaller than $M_{p}=1$. Solution of these equations shows that after a long stage of inflation the universe initially filled with the field $\phi \gg 1$ grows exponentially [1],

$$
a=a_{0} e^{\phi^{2} / 4} .
$$

If inflation begins at the Planck density $m^{2} \phi^{2} / 2 \sim 1$, the initial value of the field $\phi$ is $\sim 1 / m$. In realistic inflationary models, $m \sim 10^{-5}$, which gives the total growth of the size of the universe

$$
a=a_{0} e^{10^{10}} .
$$

This means that one can take the universe of a Planck size $10^{-33} \mathrm{~cm}$, with the Planck mass $10^{-5}$ gram, and make volume of the universe as big as $10^{10^{10}} \mathrm{~cm}$ and its mass as large as $10^{10^{10}}$ gram, give or take few orders of magnitude. After inflation, the scalar field decays, and produces a hot homogeneous universe of enormous mass.

Thus one can start with a tiny speck of space with tiny energy, no initial hot Big Bang is required, and then the process of inflation produces a huge universe like ours. This is the simplest version of the chaotic inflation scenario [2].

But inflationary universe, after enormous stretching, becomes almost exactly homogeneous, so we must explain how one can produce galaxies in this scenario. This problem was solved in 
$[3,4,5]$, where an ingenious mechanism of creation of large scale inhomogeneities due to inflationary perturbations was proposed. In this scenario, galaxies are children of quantum fluctuations produced during inflation. From this perspective, we, as children of our galaxy, are grandchildren of quantum fluctuations... This may look like a piece of science fiction. Can we test it experimentally?

\section{Can we test it experimentally?}

The best way to do it is to study properties of cosmic microwave background radiation (CMB), since the same fluctuations that produced galaxies should also produce CMB anisotropy. The properties of this anisotropy have been studies by many observers. The best and the greatest of these are WMAP and Planck satellite missions. The latest results related to inflation are summarized in the 2018 Planck 2018 data release [6].

Here are the most important predictions of inflationary cosmology, and observational data confirming them:

1) The universe must be almost exactly flat. In most models $\Omega=1 \pm 10^{-4}$. 35 years ago we only knew that $\Omega=O(1)$. The latest observational result is that $\Omega=1 \pm 10^{-2}$, in agreement with inflationary predictions. Confirmed.

2) Perturbations of the metric produced during inflation are adiabatic. Confirmed.

3) These perturbations should be gaussian. In non-inflationary models, the parameter $f_{N L}^{\text {local }}$ describing the level of the so-called local non-Gaussianity can be as large as $O\left(10^{4}\right)$, but it is predicted to be $O(1)$ in all single-field inflationary models. Prior to the Planck data release, there were rumors that $f_{N L}^{\text {local }} \sim 30$, which would rule out all or nearly all single field inflationary models. The latest results confirm that $f_{N L}^{\text {local }}=O(1)$. Confirmed.

4) Inflationary perturbations generated during a slow-roll regime with $\varepsilon, \eta \ll 1$ have a nearly flat spectrum with $n_{s}$ close to 1 . The latest result is that $n_{s} \sim 0.965$. Confirmed.

5) On the other hand, the spectrum of inflationary perturbations usually is slightly non-flat. A small deviation of $n_{s}$ from 1 is one of the distinguishing features of inflation. It is as significant for inflationary theory as the asymptotic freedom for the theory of strong interactions. Indeed, we see that the possibility $n_{s}=1$ is completely ruled out by the data. Confirmed.

6) Inflation does not produce vector perturbations. Indeed, they have not been found. Confirmed.

7) Inflationary perturbations produce specific peaks in the spectrum of CMB radiation. Indeed, many such peaks are found. Confirmed.

Thus, many general predictions of inflationary theory have been confirmed already. Moreover, observational data are precise enough to falsify many specific versions of inflationary cosmology. During the last 15 years, several classes of inflationary models have been ruled out. This includes the simplest version of chaotic inflation described above: It predicted too large level of tensor modes/gravitational waves. However, a simple modification of the theory not only restores its validity, but unifies it with several other successful versions of inflationary cosmology, in the context of a general class of $\alpha$-attractors, see [7, 8, 9] 


\section{4. $\alpha$-attractors}

There are many different ways to introduce the theory of $\alpha$-attractors. On a purely phenomenological level, the main features of inflation in all of these models can be represented in terms of a single-field toy model with the Lagrangian $[8,9]$

$$
\frac{1}{\sqrt{-g}} \mathscr{L}=\frac{R}{2}-\frac{\left(\partial_{\mu} \phi\right)^{2}}{2\left(1-\frac{\phi^{2}}{6 \alpha}\right)^{2}}-V(\phi) .
$$

Here $\phi(x)$ is the scalar field, the inflaton. The origin of the pole in the kinetic term can be explained in the context of hyperbolic geometry in supergravity and string theory. The parameter $\alpha$ can take any positive value. In the limit $\alpha \rightarrow \infty$ this model coincides with the standard chaotic inflation with a canonically normalized field $\phi$ and the inflaton potential $V(\phi)$ [2]. However, for any finite values of $\alpha$, the field $\phi$ in (4.1) is not canonically normalized. It must satisfy the condition $\phi^{2}<6 \alpha$, for the sign of the inflaton kinetic term to remain positive.

We will assume that the potential $V(\phi)$ and its derivatives are non-singular for $\phi^{2} \leq 6 \alpha$. Instead of the variable $\phi$, one can use a canonically normalized field $\varphi$ by solving the equation $\frac{\partial \phi}{1-\frac{\phi^{2}}{6 \alpha}}=\partial \varphi$, which yields

$$
\phi=\sqrt{6 \alpha} \tanh \frac{\varphi}{\sqrt{6 \alpha}} .
$$

The full theory, in terms of the canonical variables, becomes

$$
\frac{1}{\sqrt{-g}} \mathscr{L}=\frac{R}{2}-\frac{\left(\partial_{\mu} \varphi\right)^{2}}{2}-V\left(\sqrt{6 \alpha} \tanh \frac{\varphi}{\sqrt{6 \alpha}}\right) .
$$

Note that in the limit $\phi \rightarrow 0$ the variables $\phi$ and $\varphi$ coincide; the main difference appears in the limit $\phi \rightarrow \sqrt{6 \alpha}$ : In terms of the new variables, a tiny vicinity of the boundary of the moduli space at $\phi=\sqrt{6 \alpha}$ stretches and extends to infinitely large $\varphi$. As a result, generic potentials $V(\phi)=$ $V\left(\sqrt{6 \alpha} \tanh \frac{\varphi}{\sqrt{6 \alpha}}\right)$ at large $\varphi$ approach an infinitely long dS inflationary plateau with the height corresponding to the value of $V(\phi)$ at the boundary:

$$
V_{0}=\left.V(\phi)\right|_{\phi= \pm \sqrt{6 \alpha}} .
$$

To understand what is going on in this theory, let us consider, for definiteness, positive values of $\phi$ and study a small vicinity of the point $\phi=\sqrt{6 \alpha}$, which becomes stretched to infinitely large values of the canonical field $\varphi$ upon the change of variables $\phi \rightarrow \varphi$. If the potential $V(\phi)$ is nonsingular at the boundary $\phi=\sqrt{6 \alpha}$, we can expand it in series with respect to the distance from the boundary:

$$
V(\phi)=V_{0}+(\phi-\sqrt{6 \alpha}) V_{0}^{\prime}+O(\phi-\sqrt{6 \alpha})^{2} .
$$

where we denote $V_{0}^{\prime}=\left.\partial_{\phi} V\right|_{\phi=\sqrt{6 \alpha}}$.

In the vicinity of the boundary $\phi=\sqrt{6 \alpha}$, the relation (4.2) between the original field variable $\phi$ and the canonically normalized inflaton field $\varphi$ is given by

$$
\phi=\sqrt{6 \alpha}\left(1-2 e^{-\sqrt{\frac{2}{3 \alpha}} \varphi}\right)
$$


up to the higher order terms $O\left(e^{-2 \sqrt{\frac{2}{3 \alpha}} \varphi}\right)$. At $\varphi \gg \sqrt{\alpha}$, these terms are exponentially small as compared to the terms $\sim e^{-\sqrt{\frac{2}{3 \alpha}} \varphi}$, and the potential acquires the following asymptotic form:

$$
V(\varphi)=V_{0}-2 \sqrt{6 \alpha} V_{0}^{\prime} e^{-\sqrt{\frac{2}{3 \alpha}} \varphi} .
$$

Note that the constant $2 \sqrt{6 \alpha} V_{0}^{\prime}$ in this expression can be absorbed into a redefinition (shift) of the field $\varphi$. This implies that if inflation occurs at large $\varphi \gg \sqrt{\alpha}$, all inflationary predictions in this class of models of the potential $V(\phi)$ are determined only by the value of the potential $V_{0}$ at the boundary and the constant $\alpha$. For any values of $\alpha \lesssim 10$, the amplitude of inflationary perturbations, the prediction for the spectral index $n_{s}$ and the tensor to scalar ratio $r$ match observational data under a single nearly model-independent condition

$$
\frac{V_{0}}{\alpha} \sim m^{2} \sim 10^{-10} .
$$

Thus the only parameter that is required to fit the present observational data is the parameter $m \sim$ $10^{-5}$ controlling the amplitude of perturbations [10]. All such models, practically independently of the detailed properties of the inflaton potential $V(\phi)$, provide one of the best fits to the latest cosmological data [6].

\section{Universe or multiverse?}

One of the main goals of inflationary cosmology was to explain the amazing uniformity of the universe. Inflation indeed achieves this goal, but only with respect to the locally observable properties of the universe. The amplitude of inflationary perturbations grows at large scales. Already at small scales these perturbations are important enough to account for galaxy formation. At large scales, they may lead to more significant effects: They can lead to eternal inflation and to division of the universe into exponentially large parts with different laws of low energy physics operating in each of them [11]. Recent developments in inflationary cosmology, in combination with string theory, resulted in the string landscape scenario $[12,13,14]$, which asserts that our universe may be divided into exponentially many parts of exponentially large size. Each of them, from the point of view of their inhabitants, looks like a separate universe, simply because other parts of the universe are so far away that causality does not allow them to affect us. String theory suggests that there may be $10^{500}$ or even more different realizations of fundamental physical laws in different parts of the universe. This means that some of them can have large vacuum energy, and some can have small vacuum energy. But expansion in the parts with large vacuum energy is so fact that it would tear galaxies apart. Thus we can live only in the parts of the universe with a tiny vacuum energy, consistent with its presently measured value. This provides the best presently known solution of the cosmological constant problem.

Similarly, we can live only in the parts of the universe where the electron mass is small, and its charge is similar to its presently measured value. We can live only in the parts where the masses of the proton and neutron are very similar to each other, and when the number of space-time dimensions is four. All of these facts have been known to us for many years, they constituted the basis of the anthropic principle, but prior to the development of the theory of inflationary multiverse and string theory landscape it was difficult to make any sense of it. The standard idea was that there 
is only one universe, only one value of the electron mass and of the vacuum energy, so there is no choice. This picture has now changed, perhaps irreversibly. Everyone knows that ice and water have the same chemical composition, but fish cannot live in ice.

Thus, attempts to revisit the basic cosmological principles, gradually moved us towards the theory of inflationary universe, and then to the theory of the multiverse, which changes our views on the origin and global structure of our world. The emerging picture is certainly incomplete, and its proper interpretation will require full development of quantum cosmology, in combination with string theory.

\section{References}

[1] A. D. Linde, "Particle physics and inflationary cosmology," Contemp. Concepts Phys. 5, 1 (1990) [hep-th/0503203].

[2] A. D. Linde, "Chaotic Inflation," Phys. Lett. 129B, 177 (1983).

[3] V. F. Mukhanov and G. V. Chibisov, "Quantum Fluctuation And 'Nonsingular' Universe," JETP Lett. 33, 532 (1981) [Pisma Zh. Eksp. Teor. Fiz. 33, 549 (1981)].

[4] S. W. Hawking, "The Development Of Irregularities In A Single Bubble Inflationary Universe," Phys. Lett. B 115, 295 (1982); A. A. Starobinsky, "Dynamics Of Phase Transition In The New Inflationary Universe Scenario And Generation Of Perturbations," Phys. Lett. B 117, 175 (1982); A. H. Guth and S. Y. Pi, "Fluctuations In The New Inflationary Universe," Phys. Rev. Lett. 49, 1110 (1982); J. M. Bardeen, P. J. Steinhardt and M. S. Turner, "Spontaneous Creation Of Almost Scale - Free Density Perturbations In An Inflationary Universe," Phys. Rev. D 28, 679 (1983).

[5] V. F. Mukhanov, "Gravitational Instability Of The Universe Filled With A Scalar Field," JETP Lett. 41, 493 (1985) [Pisma Zh. Eksp. Teor. Fiz. 41, 402 (1985)]; V. F. Mukhanov, Physical Foundations of Cosmology, Cambridge University Press, 2005.

[6] Y. Akrami et al. [Planck Collaboration], "Planck 2018 results. X. Constraints on inflation," arXiv:1807.06211 [astro-ph.CO].

[7] R. Kallosh, A. Linde and D. Roest, "Superconformal Inflationary $\alpha$-Attractors," JHEP 1311, 198 (2013) [arXiv:1311.0472 [hep-th]].

[8] M. Galante, R. Kallosh, A. Linde and D. Roest, "Unity of Cosmological Inflation Attractors," Phys. Rev. Lett. 114, no. 14, 141302 (2015) [arXiv:1412.3797 [hep-th]].

[9] R. Kallosh and A. Linde, "Escher in the Sky," Comptes Rendus Physique 16, 914 (2015) [arXiv:1503.06785 [hep-th]].

[10] R. Kallosh and A. Linde, "Planck, LHC, and $\alpha$-attractors," Phys. Rev. D 91, 083528 (2015) [arXiv:1502.07733 [astro-ph.CO]].

[11] A. D. Linde, "Eternally Existing Self-Reproducing Chaotic Inflationary Universe," Phys. Lett. B 175, 395 (1986).

[12] S. Kachru, R. Kallosh, A. D. Linde and S. P. Trivedi, "De Sitter vacua in string theory," Phys. Rev. D 68, 046005 (2003) [hep-th/0301240].

[13] M. R. Douglas, “The Statistics of string / M theory vacua," JHEP 0305, 046 (2003) [hep-th/0303194].

[14] L. Susskind, "The Anthropic landscape of string theory," In *Carr, Bernard (ed.): Universe or multiverse?* 247-266 [hep-th/0302219]. 